\title{
Maltrato al adulto mayor institucionalizado - una revisión sistemática
}

\section{Institutionalized maltreatment to the elderly adult - a systematic review}

\author{
Nataly Londoño $\mathrm{Q}^{1} \mathbb{1}^{\mathbb{D}}$; Miguel A Cubides $\mathrm{T}^{1}$ (i) \\ Forma de citar: Londoño N, Cubides M. Maltrato al adulto mayor institucionalizado- una revisión sistemática. Salud UIS. 53: \\ e21022. doi: https://doi.org/10.18273/saluduis.53.e:21022 @) ()
}

\section{Resumen}

Introducción: el maltrato al adulto mayor va en incremento y se identifica de distintas formas. Objetivo: conocer el panorama del maltrato al adulto mayor institucionalizado y a partir de una revisión sistemática de literatura, identificar causas, tipos, principales características y signos diagnósticos de violencia a esta población, en instituciones prestadoras de servicio de salud o alojamiento para ellos. Metodología: revisión de literatura internacional en español e inglés sobre el maltrato del adulto mayor en diferentes instituciones, en las bases de datos: Scielo, Medgraphic literatura biomédica, Redalyc, Science direct, Pubmed, Scopus, Dialnet, Base y Redib, bajo la metodología PRISMA. Resultados: se revisaron 301 referencias que muestran incidencia del maltrato al adulto mayor institucionalizado, características y consecuencias, esto permitió hacer un balance de las causas, tipos, signos y síntomas del maltrato que frecuentemente se presentan. Conclusión: el maltrato en ancianos es un problema de salud pública y social que deja graves consecuencias en la calidad de vida del adulto mayor y representa un reto para la atención en salud.

Palabras clave: Anciano; Maltrato; Violencia; Salud; Asilo; Cuidadores; Organizaciones.

\begin{abstract}
Introduction: Elder abuse is increasing and it is identified in different ways. Objective: To learn about the panorama of institutionalized elder mistreatment from a systematic literature review, identify causes, types, main characteristics, and diagnostic signs of violence in this population, in institutions which provides health services or housing for them. Methodology: Review of international literature in English and Spanish on elder abuse in different institutions in the following data bases: Scielo, Medgraphic biomedical literature, Redalyc, Science direct, Pubmed, Scopus, Dialnet, Base and Redib, under the PRISMA methodology. Results: A review of 301 references show the incidence of institutionalized elder abuse, characteristics, and consequences. This permitted a balance of the causes, types, signs, and symptoms of mistreatment that frequently occur. Conclusion: Elder abuse is a public and social health problem that has serious consequences on the quality of life of the elderly and represents a challenge for health care.
\end{abstract}

Keywords: Elderly; Abuse; Violence; Health; Asylum; Caregivers; Organizations.

1. Universidad de Caldas. Manizales, Colombia.

Correspondencia: Nataly Londoño Quintero. Dirección: Cra 7ª \# 15a-26, Caldas. Teléfono: +57 3128814726. Correo electrónico: quinteronataly069@gmail.com 


\section{Introducción}

Investigaciones recientes como: "Riesgo de maltrato en personas adultas mayores: validación de contenido de una escala", publicado en enero de 2019 ; "Elder abuse, a silent problem", revisión sistemática publicada en agosto de 2020; "Maltrato de las personas mayores", reporte de la OMS de junio de 2020 y reportes publicados constantemente por el equipo Elderabuse.org; muestran la realidad del incremento de casos de maltrato a la población de adultos mayores a nivel mundial, brindan un panorama amplio acerca de las características y consecuencias de cada tipo de maltrato, y nos plantean un reto en el campo de la salud pública para enfrentar esta problemática social. Teniendo en cuenta estos antecedentes, en este artículo se desarrolla el objetivo de conocer el panorama en el ámbito del maltrato en el adulto mayor institucionalizado a partir de una revisión de literatura, con énfasis en aspectos como causas, tipos, principales características y signos diagnósticos de violencia a esta población, en ambientes específicos como lo son instituciones prestadoras de servicio de salud o alojamiento para ancianos.

Se puede decir que la población mundial está envejeciendo aceleradamente. En muchos países se ha encontrado un incremento en la esperanza de vida y se proyecta que la proporción de los habitantes mayores de 60 años se duplique para el 2050, pasando del $11 \%$ al $22 \%$ del total de la población ${ }^{1}$. El abandono en el adulto representa una problemática social que aumenta por los cambios en la natalidad y mortalidad de los últimos tiempos, evidenciando el gran reto que se encuentra en transformar la atención y la protección de la población adulta mayor.

El alojamiento de los adultos mayores en asilo o casa de reposo es consecuencia de las diferentes situaciones como: maltrato físico, emocional, psicológico y abandono familiar ${ }^{2}$. El maltrato es una situación que se ha presentado por mucho tiempo alrededor del mundo y ha sido clasificado de diferentes formas ${ }^{3,4}$. Los estudios más recientes en este tema, invitan a ver a la población adulta como un grupo de personas vulnerables al trato inadecuado, al abandono y a la soledad; para así enfrentar esta problemática aportando ideas y acciones que incrementen el bienestar de todos ellos $^{5}$. Actualmente, hay evidencia de que en varias instituciones residenciales o de cuidado transitorio para el adulto mayor se presentan casos de maltrato para esta población, situaciones que se multiplican por la falta de conocimiento acerca de las características y realidades del maltrato y la indiferencia social y política ante esta situación ${ }^{2,4,5}$.

\section{Materiales y métodos}

La presente investigación se realiza a partir de una búsqueda de literatura, en español e inglés, sobre el maltrato al adulto mayor institucionalizado. El proceso de búsqueda se llevó a cabo desde marzo de 2018 hasta enero de 2020, se usó el método de búsqueda por palabras clave verificadas en tesauros con los descriptores: adulto mayor, maltrato, violencia, institucionalizado y asilo, (Older adult, abuse, violence, institutionalized, asylum), con los conectores AND, OR. Se utilizaron las bases de datos: Scielo, Medgraphic literatura biomédica, Redalyc, Science direct, Pubmed, Scopus, Dialnet, Base, Redib y otras fuentes como Universidad de Chile, Universidad Nacional, Universidad Técnica del Norte, la página Elderabuse.org y el Servicio de información de discapacidad. Se tuvieron en cuenta como criterios de inclusión: Periodo de publicación, se consideraron sólo estudios realizados entre el 2015 y el 2020 (5 años de anterioridad); Características del estudio, se acudió a meta análisis, revisiones sistemáticas, revisiones teóricas, estudios aleatorizados y opinión de expertos divulgados en medios científicos; Idioma, castellano e inglés; y como Criterios de exclusión: estudios que en el título indicaban maltrato doméstico, obteniendo un total de 3270 artículos científicos y 15 documentos de interés.

Se realizó con las indicaciones PRISMA para verificar una revisión transparente y fiable y se utilizó una hoja electrónica de EXCEL para la organización de los datos obtenidos, teniendo en cuenta las variables año de publicación, país, idioma, resumen, causas, tipos y consecuencias del maltrato. Después de realizar la sistematización de la información se eliminan los artículos repetidos, identificados por el título, y quedan un total de 1876 , de estos se excluyen 1428 por ser documentos que no aportaban datos importantes en cuanto a los tipos, consecuencias o causas del maltrato, sino que trataban temas de maltrato doméstico, violencia intrafamiliar y situaciones conflictivas con cuidadores informales; temas identificados a partir del resumen de los artículos y que no eran relevantes para la revisión. Después de la exclusión se obtienen un total de 448 artículos y se revisan 301 por ser los artículos que se encuentran en texto completo. Una vez terminada la revisión, se considera importante incluir 54 artículos dentro de las citas de esta revisión por tratar directamente los temas de interés para esta revisión y aportar información importante para el análisis, se seleccionan por ser artículos que tratan de forma específica el tema de maltrato institucional y exponen panoramas amplios de las características, consecuencias e implicaciones del 
maltrato para el adulto mayor. Los artículos que fueron excluidos no aportaban datos nuevos o relevantes. Después de la evaluación por pares, se decide incluir en la revisión otras 4 referencias que aportan datos de interés en cuanto al panorama global del maltrato institucional al adulto mayor. Figura 1.
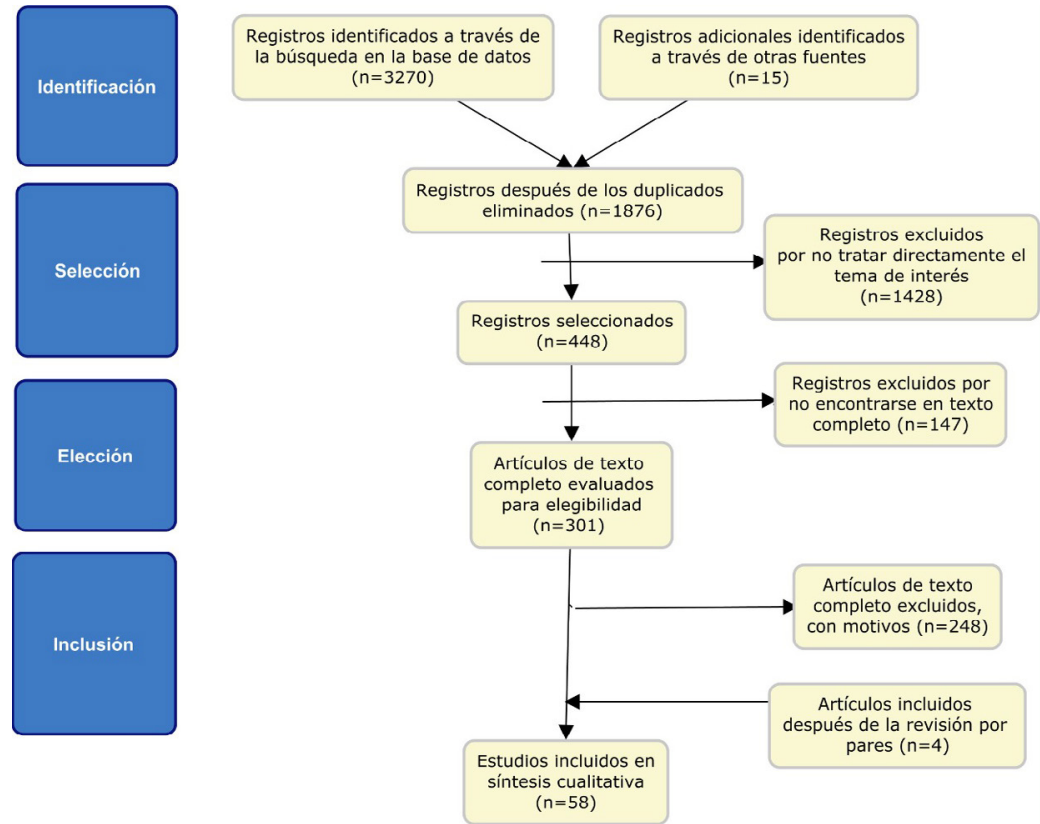

Figura 1. Flujograma del modelo PRISMA para la selección de los artículos.

\section{Resultados}

La Organización Mundial de la Salud (OMS) define al maltrato del adulto mayor como “...la acción única o repetida, o falta de la respuesta apropiada, que ocurre dentro de cualquier relación donde exista una expectativa de confianza y la cual produzca daño o angustia a una persona adulta..." ${ }^{\prime 6}$. El maltrato del adulto mayor también se constituye como una dificultad en la atención institucional a nivel mundial, lo cual desencadena alteración en su salud física y psicológica. Un agravante de la situación de maltrato es el aumento progresivo con el paso del tiempo, lo que representa un desafío de salud pública en cualquier país sin importar culturas o economías ${ }^{7,8}$.

\section{Factores de riesgo:}

- Factores de riesgo del anciano: factores como deterioro de la salud, incontinencia, edad avanzada, dependencia física, alteraciones mentales, maltrato previo y aislamiento social ${ }^{6,9}$.

- Enfermedades de base: el adulto mayor tiene una alta posibilidad de desarrollar patologías debilitantes, incapacitantes o terminales, exponiéndolo en situaciones de vulnerabilidad frente al maltrato; es por esto que requieran de atención personalizada y de tiempo completo para suplir sus necesidades. En este grupo de patologías encontramos todas aquellas de carácter crónico y degenerativo, que dejan como consecuencia algún grado de dependencia física o la muerte.

- Dependencia: la dependencia del adulto mayor puede verse reflejada en 3 formas: física, económica y emocional. La física se puede entender como la dificultad o incapacidad para ejecutar actividades de la vida diaria. Esta incapacidad se genera por el proceso deletéreo que viene ligado a la edad; sin embargo, "...el proceso de envejecimiento no es sinónimo de enfermedad, sino que, el adulto mayor; es vulnerable a padecer enfermedades sobre todo crónicas, que conducen fácilmente a la morbilidad, discapacidad y dependencias"3.

- Factores de riesgo del cuidador: algunas situaciones que exponen a la persona cuidadora a ejercer maltrato al adulto mayor incluyen; sensación de estrés, sobrecarga física, emocional y psicológica. Patologías resultantes de esfuerzos excesivos, trastornos psicopatológicos, abuso de sustancias, vivencias de violencia familiar, falta de 
contacto social, antecedentes de abuso económico de parte del victimario, apoyo insuficiente para el cuidado del anciano, múltiples obligaciones conyugales, paternas, domésticas o laborales y ser hombre ${ }^{6,10}$.

\section{Causas:}

En la actualidad, se identifican múltiples causas de maltrato al adulto mayor, todas relacionadas con ejercer control sobre la persona dependiente, marcado por:

- Carencia de educación y cultura sobre el proceso deletéreo que representa envejecer.

- Ausencia de programas de promoción de derechos.

- Carencia de estrategias para la detección temprana de maltrato.

- Ausencia de denuncias.

- Estrategias poco eficientes en la protección del adulto mayor.

- Falta de respeto y de agradecimiento de las poblaciones al adulto mayor.

- Sobrecarga de los cuidadores, enfermeras y demás personal de salud ${ }^{3}$.

\section{Tipos de maltrato:}

- Maltrato físico: empleo de fuerza física para generar lesión, dolor o discapacidad al anciano $^{4}$ como: golpes, quemaduras, fracturas, administración abusiva de fármacos o tóxicos ${ }^{6}$.

- Maltrato psicológico: generar perjuicio, dolor o castigo mediante agresión verbal, intimidación, austeridad, engaño, y producir incomodidad psicológica ${ }^{4}$; produce sensación de alta fragilidad, debilitamiento de autoestima, irrupción en la intimidad, despersonalización y aumento de la situación de soledad ${ }^{3}$.

- Maltrato emocional: omisión de muestras de cariño y fallas en la comunicación asertiva ${ }^{4}$.

- Abandono: acción de desentendimiento sin razón aparente arriesgando el bienestar de la persona sobre la cual se es responsable legalmente ${ }^{4}$.

- Abuso sexual: contacto o acciones sexuales sin consentimiento $^{4}$, se evidencia de manera abierta o se esconde tras expresiones faciales, comunicaciones verbales, insinuaciones, caricias, exposición de genitales o violación ${ }^{3}$.

- Negligencia: acción de olvido, en la que se suspende el suministro de elementos para la atención básica del adulto mayor ${ }^{4}$, incluye falta de cuidados higiénicos, vivienda, alimentos, seguridad y tratamientos médicos, y puede ser activa o pasiva. La negligencia activa hace referencia a la ausencia de atenciones necesarias hacia el adulto mayor dependiente por parte del cuidador principal a causa de descuido o apatía, es considerada como un acto deliberado, en tanto que la negligencia pasiva es considerada como un acto involuntario que resulta del desconocimiento o incapacidad para proveer cuidados necesarios al anciano ${ }^{6}$.

- Abuso económico: acción jurídicamente demostrable e inadecuada de aprovechamiento económico y/o la utilización de recursos o fondos financieros, específicamente, obstaculizar uso de dinero por parte del propietario, extorsión económica o desmán patrimonial ${ }^{4,11}$.

- Maltrato invisible: la escasa, o en ocasiones nula visibilidad de los malos tratos en los lugares de estadía para el adulto mayor, es la mayor razón de subvaloración. Esta modalidad de maltrato se ejecuta con la utilización de técnicas de coerción, alterar la vida digna y limitar la autonomía para la toma de decisiones. Se evidencia, además, con una atención insuficiente o de mala calidad, que desencadena complicaciones para las diferentes patologías y estado general que maneje el adulto mayor. Una muestra clara de este tipo de maltrato es la incorrecta administración de medicamentos, incumplir con sus horarios u omitir el suministro de los mismos, cuando no se facilitan las condiciones ambientales óptimas o se lanzan comentarios hostiles, cuando se irrumpe la privacidad, se establecen restricciones físicas sin necesidad evidente y no se consideran los obstáculos arquitectónicos que desencadenan caídas ${ }^{12}$.

- Ensañamiento terapéutico: se produce cuando se realizan procedimientos diagnósticos molestos o dolorosos que podrían ser innecesarios, pero a su vez, también el mantener tratamientos que afectan de forma negativa a la calidad de vida del paciente, prolongando una situación irreversible o terminal ${ }^{13}$.

- Maltrato farmacológico: se lleva a cabo mediante el uso excesivo de calmantes cuando no es necesario o negación de la administración de los mismos cuando lo precisan ${ }^{13}$.

- Restricciones: tanto físicas como químicas, ya que limitan de toda capacidad de movimiento libre a la persona $^{13}$.

Categorías de maltrato en las instituciones de adultos mayores: se establecen cuatro categorías: la infantilización, cuando se ofrece al adulto mayor un trato que normalmente se le da a un niño que todavía no tiene la responsabilidad suficiente y al que no se le pueden confiar tareas o que haceres; la despersonalización, cuando no se consideran las condiciones o necesidades 
específicas de cada paciente y se ofrece una atención generalizada; la deshumanización, consiste en ignorar al anciano, negarle su privacidad y quitarle cualquier responsabilidad que esté en condiciones de asumir; y por último, la victimización, en donde se interrumpe la integridad tanto física como psicológica del adulto mayor, por medio de ofensas, penalizaciones físicas, intimidaciones, entre otras ${ }^{14}$. Estas modalidades de maltrato ocurren en una gran parte de la población adulta mayor, con mayor frecuencia en el género femenino, en el rango de 60 a 74 años ${ }^{14}$.

\section{Situaciones clínicas en el anciano sospechoso de maltrato: (Diagnóstico)}

- "Períodos de tiempo prolongados entre la aparición de una lesión o patología y la solicitud de asistencia.

- Incongruencias en el relato de los hechos cuando ocurre un accidente, discrepancias entre las versiones del cuidador y el paciente.

- Explicaciones contradictorias y poco creíbles acerca de la manera como se origina el incidente.

- Visitas recurrentes a los servicios de urgencias o necesidad repetida de solicitar atención médica por complicaciones de enfermedades de base, pese a contar con un adecuado plan de manejo en casa o institución de alojamiento.

- Identificación al momento de la consulta médica de un adulto mayor en condiciones de deterioro físico, funcional o cognitivo sin contar con la compañía y el respaldo de su cuidador principal.

- Cumplimiento débil e insuficiente del tratamiento médico, con dosis alteradas (por exceso o por defecto) o consumo de fármacos (psicótropos) no prescritos por el personal médico autorizado.

- Dificultad para conseguir una comunicación asertiva con el adulto mayor cuando está presente el cuidador.

- Antecedentes de episodios que sugieren ser víctima de maltrato" $"$.

\section{Señales de alerta, signos y síntomas de maltrato:}

- "Justificaciones sin lógica aparente acerca de la forma como se producen los accidentes o las lesiones.

- Demoras para acudir a los servicios de asistencia médica.

- Visitas frecuentes a servicios de salud por razones cambiantes.

- Administración incorrecta de medicamentos.

- Falta de respuesta eficiente para los tratamientos propuestos.
- Lesiones en la piel (úlceras) con una mala evolución, a pesar de aplicar las medidas adecuadas de tratamiento.

- Desnutrición importante sin razón aparente.

- Deshidratación.

- Caídas frecuentes.

- Actitud de temor, ansiedad o pasividad.

- Negativa fuerte a dejar solo al anciano por parte del cuidador"15.

\section{Consecuencias:}

- Físicas: hematomas, equimosis, contusiones, fracturas de diferentes tipos, quemaduras de diferentes grados, abrasiones, laceraciones, arañazos, heridas por ataduras, luxaciones ${ }^{13}$.

- Psicológicas: confusión, indefensión, agitación, pseudodemencias, ideas e intentos suicidas, aislamiento social, emocionalmente deprimido o ansioso, pérdida de apetito, temor y alteraciones del sueño ${ }^{13}$.

- De negligencia: exacerbación de enfermedades de base, ulceras por presión, hipotermia, hipertermia, deficiente higiene corporal, malnutrición, desnutrición y deshidratación ${ }^{13}$.

- Económicas: nivel de vida deteriorado por la disminución o supresión de los ingresos, falta de servicios básicos, incapacidad para pagar sus servicios públicos, alteración de firmas, modificaciones en los hábitos de ingresos y egresos y desahucio ${ }^{13}$.

- Sexuales: enfermedades de transmisión sexual, traumas o lesiones en áreas genitales, pechos, boca o zona anal y hemorragias vaginales ${ }^{13}$.

Entre las problemáticas de salud de interés, la población adulta mayor tiene muy poca representación. Sin embargo, actualmente esta población registra un aumento epidemiológico que expone la importancia y relevancia de su situación ante investigadores ${ }^{16}$. El proceso de envejecimiento de una población surge como producto de las dinámicas demográficas y epidemiológicas de la actualidad, el descenso en las tasas de mortalidad y natalidad, y elevación importante de la esperanza de vida ${ }^{17}$. Alrededor del mundo, se reporta que para el periodo comprendido entre los años 2000-2050, la población igual o mayor de 60 años se duplicará, y pasará del $11 \%$ al $22 \%$ de la población mundial $^{1}$. Todas las circunstancias anteriormente expuestas, se han convertido en factores desencadenantes de la institucionalización de la población senil $^{18}$. El proceso de envejecimiento demográfico de la población desencadena grandes retos y modificaciones en las estructuras sociales, económicas y culturales. 
Estos cambios demográficos involucran, a su vez, la imperiosa necesidad de contar con una buena preparación, para garantizar condiciones óptimas de vida para una sociedad en envejecimiento. Es de esta manera, como se destaca la importancia de la implementación de actividades de promoción y prevención, con la meta de eliminar la exclusión y los malos tratos que se constituyen como producto de estereotipos que se mantienen a través de los años, pasando de generación en generación, a raíz del distanciamiento entre generaciones y la falta de programas de educación y protección ${ }^{19}$.

Existen desafíos para la atención al adulto mayor que deben tenerse en cuenta en el proceso de envejecimiento, sobre todo los que tienen relación con el crecimiento demográfico y su estado de salud, ya que en caso de requerir atención y cuidados especializados aumentan los costos. Esto, en cierta medida puede significar debilitamiento de los recursos económicos y de apoyo (social o familiar), lo cual afecta la labor de proveer y mantener una adecuada calidad de vida para el adulto mayor ${ }^{20}$. Resulta inevitable identificar la necesidad de impulsar la buena atención al adulto mayor en todas sus facetas, para lo que se hace necesario reconocerque “... la gerontogeriatria comunitaria es una oportunidad que vinculada a las diferentes especialidades de la salud, como la psiquiatría, la psicología, la neurología y la medicina interna... " ${ }^{21}$, y genera un ambiente favorable para la prestación de servicios de salud oportuna desde que inicia el proceso de envejecimiento ${ }^{22}$.

Entre las principales actividades de prevención y detección temprana del maltrato al adulto mayor se encuentran: aumento y vigilancia de la disponibilidad y uso de recursos destinados al cumplimiento de las leyes, tamizaje de abuso a la población adulta mayor, reforzamiento en la difusión de información y sensibilización de las personas sobre el fenómeno del maltrato, educación y capacitación constante $\mathrm{y}$ de calidad al personal de salud y a la población de cuidadores principales ${ }^{9}$. Además, el fortalecimiento de las relaciones familiares, ya que un apoyo familiar insuficiente, genera en el anciano asilado una alta probabilidad de desarrollar complicaciones en su salud física, psicológica o emcional ${ }^{23,24}$.

"Actualmente, el maltrato al adulto mayor representa un importante problema para la salud pública y social, ya que es una situación que repercute en todas las generaciones y de la que, todo el núcleo familiar y generacional es responsable" ${ }^{25}$.
"El abuso al adulto mayor no es un fenómeno emergente, sin embargo, en muchas ocasiones se encuentra oculto, con actividades de detección muy débiles que no logran identificarlo y que pueden ser consecuencia de la falta de normatividad como guías y protocolos de detección, así como a la ausencia de concientización e indagación por parte del profesional de la salud encargado o miedo de generar reportes por parte de la persona agredida" ${ }^{26,27}$.

Finalmente, en la citación del trabajo "Envejecimiento activo: un marco político", OMS $2002^{31,32}$ encontramos que "la agresión al adulto mayor significa por ende una violación de los derechos humanos $y$ un desencadenante importante de complicaciones para su salud"33. Para enfrentarse al problema del maltrato con el adulto mayor y tratar de reducirlo se requiere un trabajo multisectorial y multidisciplinario, que reconozca que las actividades de sensibilización y detección temprana del maltrato son los puntos a priorizar en el desarrollo de las labores de institucionalización $^{34,35}$. Se considera que estas herramientas son fundamentales para los profesionales de la salud, y en general para todos los profesionales que conforman el equipo de trabajo ${ }^{36}$. "Es de vital importancia realizar una identificación del maltrato oportuna y eficiente, de igual manera que la promoción e implementación de atenciones individualizadas y de calidad"37, lo cual se traduce esencialmente en un buen nivel de conocimiento de los cuidadores sobre el manejo del adulto mayor en general ${ }^{38-40}$.

\section{Discusión}

Las instituciones de alojamiento para adultos mayores son espacios donde la persona reside por periodos de tiempo prolongados y se le provee un nivel de bienestar óptimo y cuidados básicos ajustados a sus necesidades ${ }^{41}$. Sin embargo, el adulto mayor que se encuentra institucionalizado se caracteriza por un debilitamiento o supresión de las relaciones sociales y familiares, enmarcado en la teoría de desvinculación social, la cual expone una convergencia de varios factores como, alejamiento de conexiones personales, pérdida del papel laboral, disminución de la comunicación con la familia y amigos y traslado de su vivienda ${ }^{42}$.

"La situación del maltrato no es algo nuevo. Es una realidad que se ha mantenido a través de muchos años, desde las más antiguas civilizaciones, lo que permite inferir que trae implícito un factor cultural importante, que propicia que este fenómeno perdure a lo largo de la 
historia de la humanidad"10. "Una expectativa de vida cada vez más elevada, impone grandes retos para la atención sanitaria, el sistema de salud, la distribución y manejo de los recursos económicos, la preparación de los profesionales de la salud y cuidadores y la asistencia social y comunitaria"12, por lo que "se recomienda profundizar en esta temática para que los entes correspondientes puedan elaborar estrategias de control y evitarlo al máximo" ${ }^{43}$.

Situación similar a la encontrada en esta revisión se observan en las cifras de la OMS (2014), donde se evidencia que "entre un $4 \%$ y un $6 \%$ de los adultos mayores, afirman ser víctimas de malos tratos considerables en el último mes"26. En este grupo etario, "la situación de maltrato se puede exacerbar con el aumento de la longevidad, la cual suele acompañarse de una elevación o complicación de patologías físicas y patológicas y, comúnmente algún grado de discapacidad física o mental. Los adultos mayores que presentan algún grado de discapacidad ya sea física o mental, poseen un riesgo mayor por tener limitaciones propias de las actividades de la vida diaria que los expongan a una situación de dependencia parcial o total. Separar este párrafo resistencia física para defenderse y protegerse de los actos violentos, la limitada capacidad para huir de ésta y/o mayor dificultad para comprender y denunciar cualquier tipo de abuso son algunas explicaciones atribuibles para el riesgo de abuso en adultos mayores con discapacidades" "44. "Los factores que predisponen a la persona cuidadora a ejercer tratos violentos a un anciano incluyen la sensación de estrés, deficiente salud mental, presencia de alguna psicopatología, abuso de sustancias como alcohol y drogas, antecedentes de dependencia financiera, falta de una red apoyo fuerte para el cuidado del anciano y ser hombre"45. La atención de salud al adulto mayor institucionalizado debe buscar estrategias que faciliten una atención integral, además de contar con la cooperación de los usuarios, el personal de salud y los familiares directamente responsables ${ }^{46}$, fenómeno comparable con el estudio financiado por la OMS publicado en la revista The Lancet Global Health.

Entre las controversias y retos médicos se incluye el maltrato a la población anciana o adulta mayor ${ }^{17}$. A través de las entrevistas se puede identificar que el proceso de envejecer es particular y complejo para cada individuo, también se aprecian diferentes perspectivas sobre la vejez y su camino por ella ${ }^{47}$. A medida que aumentan los índices de envejecimiento, se elevan los riesgos de dependencia por deterioro funcional, lo cual genera costos elevados en los ámbitos individuales, familiares y sociales ${ }^{48,49}$. "Es por esto que se considera fundamental estudiar, analizar la temática de la vejez. No se debe permanecer ajeno a esta realidad que se encuentra marcada por el envejecimiento demográfico, siendo de suma importancia analizar cómo los adultos mayores vivencian la vejez, teniendo en cuenta en este documento que se trata de una vejez particular, la vejez institucionalizada" ${ }^{50}$.

En la actualidad, los adultos mayores proveen la mayor expresión de diversidad de la historia de la humana, "las principales divergencias radican en el nivel socioeconómico, ambiente y relaciones familiares, estado de la salud y acceso a los servicios sanitarios, antecedentes personales y profesionales de cada persona, e influencia de la cultura" ${ }^{51}$.

El PLANPAM 2013-2017 se refiere al envejecimiento saludable como "la mejoría en el nivel de bienestar físico, psíquico y social de la población anciana se consigue por medio del aumento en la cobertura y calidad de los servicios socio sanitarios, la garantía de una asistencia alimentaria y nutricional, la oferta de oportunidades para una vida armónica dentro de su núcleo familiar y su comunidad" 52 . Como resultado de esta revisión podemos observar que cuando los servicios sanitarios se alejan de contribuir a ese envejecimiento saludable, se favorecen situaciones y ambientes de maltrato de diferentes tipos.

Adams, Sanders y Auth (2004), evaluaron en su estudio la relación entre la soledad y las expresiones depresivas en 163 adultos mayores institucionalizados en centros de asistencia y alojamiento ${ }^{53}$, situación que coincide con lo encontrado en esta revisión al exponer el abandono como tipo de maltrato que deja consecuencias psicológicas en los adultos mayores.

La facultad de Ciencias Jurídicas y Sociales de la Universidad de Talca, concluye en el estudio publicado sobre el maltrato al adulto mayor ${ }^{54}$ que este es una problemática de importancia e interés social y a pesar de todo sigue siendo invisibilizado. Dicha situación se desencadena por múltiples causas y se manifiesta en diferentes ámbitos, tales como el sociocultural, el jurídico y el económico. En el primero de ellos, la importancia radica en el binomio entorno-persona mayor. Las consecuencias más letales en este ámbito son las posibilidades de aislamiento, la disminución de autoestima y las emociones de inseguridad que favorecen la creación de estereotipos negativos para la vejez, hecho con el que coincidimos en nuestra investigación, ya que encontramos en la literatura consultada los mismos tipos de maltrato y las mismas consecuencias. 


\section{Conclusiones}

El maltrato en ancianos es un problema de salud pública que deja graves consecuencias en la calidad de vida del adulto mayor y representa un reto para la atención en salud. Las instituciones prestadoras de servicios para los adultos mayores deben ser vigiladas por los entes correspondientes para prevenir, detectar y detener las situaciones de maltrato.

Cualquier tipo de maltrato al que se vean expuestos los adultos mayores deja secuelas en su vida que lo reducen e imposibilitan para ejecutar con normalidad las actividades de la vida diaria.

El proceso de envejecimiento trae consigo debilitamiento físico y mental, lo cual expone al adulto mayor a presentar mayor vulnerabilidad de ser maltratado. Las situaciones de dependencia son un factor de riesgo importante para recibir maltrato por ser una situación que demanda mayor atención de parte del cuidador, quien debe dedicar más tiempo y esfuerzo en sus cuidados, esto muchas veces se traduce en desgaste y termina maltratando al adulto mayor.

El personal de salud encargado del cuidado del adulto mayor debe estar capacitado en este grupo poblacional; deben ser personas que cuenten con experiencia, buena disposición y actitudes que garanticen el buen trato al adulto mayor; los horarios de trabajo no deben ser exhaustivos y cada institución debe contar con el personal suficiente para no generar cargas de trabajo en ningún empleado.

\section{Referencias}

1. Melendez Herrera MC, Robalino Quintana MA. Repercusión del abandono en el estado mental y emocional del adulto mayor institucionalizado en los asilos de ancianos Sagrado Corazón de Jesús de la ciudad de Ambato y el asilo de ancianos de Patate. [Tesis para optar al título de médico]. Ecuador. Universidad Técnica de Ambato; 2015.

2. Toribio Ferrer C, Franco Barcenas S. Percepción de los adultos mayores acerca de sus vivencias en una casa de reposo. Rev Enferm Inst Mex Seguro Soc. 2018; 26(1): 16-22.

3. Condori Barrera JR. Causas, consecuencias de maltrato en el adulto mayor. Repositorio unas. 2015; 18(24): 2-4.

4. Rodríguez-Lombana, L., \& Chaparro-Díaz, L. Soporte social y sobrecarga en cuidadores. Rev CUIDARTE. 2020. doi: https://doi.org/10.15649/ cuidarte. 885
5. Pabon Poches DK, Delgado Meza JA. Maltrato en la población adulta mator: una revisión. Cuaderno Venezolano de sociología. 2017; 26(2): 245-267.

6. Cano SM, Garzón MO, Segura M, Cardona D. Factores asociados al maltrato del adulto mayor de Antioquia. Rev Fac Nac Salud Pública. 2015; 33(1): 67-74.

7. Abata Erazo AP, Guijarro Revelo VA. Sobrecarga del cuidador relacionado al maltrato del adulto mayor en usuarios del servicio de consulta externa del hospital Luis Gabriel Dávila de la ciudad de Tulcán, durante el período de septiembre a noviembre del 2016. Puce. 2016; 18(24): 2-4.

8. Jiménez Aguilera B, Baillet Esquivel LE, Avalos Perez F, Campos Aragón L. Dependencia funcional $\mathrm{y}$ percepción de apoyo familiar en el adulto mayor. Aten Fam. 2016; 23(4). doi: https://doi. org/10.1016/j.af.2016.08.002

9. Cano SM, Garzón MO, Segura AM, Cardona D. Factores asociados al maltrato del adulto mayor de Antioquia, 2012. Rev. Fac. Nac. Salud Pública. 2015; 33(1): 67-74.

10. Pabón-Poches DK. Riesgo de maltrato en personas adultas mayores: validación de contenido de una escala. Psicogente. 2019; 22(41): 1-27. doi: https:// doi.org/10.17081/psico.22.41.3299

11. Montero Solano G, Vega Chaves JC, Hernández G. Abuso y Maltrato en el Adulto Mayor. Medicina. Pierna. Costa Rica. 2017; 34(1): 120-130.

12. Hernández Verdecía I, Rodríguez Hernández D, Repilado Rodríguez L. El adulto mayor como víctima desde la victimología. Protección jurídica de sus derechos en cuba. Univ Ciencia. 2019; 8(1): 175-186.

13. Serra Valdés M, Cordero López G, Viera García M. El maltrato a los ancianos o el no mejor trato. Realidad y retos. Medisur. 2018; 16(2): 233-240.

14. Loez López R. fenómeno invisible: maltrato en los ancianos. Universidad de Cantabria. 2017.

15. Gallo Durán R, Díaz Gutiérrez Y. Factores asociados al maltrato y violencia en el adulto mayor. Universidad Wiener. 2016; 22(1): 25-27.

16. Moreno M. Detección y abordaje de la sospecha de maltrato al anciano institucionalizado. Universidad de Valladolid. 2015.

17. Laguado JE, Camargo Hernández KC, Campo Torregroza E, Martín Carbonell MC. Functionality and degree of dependence on elderly home care centers in welf. Gerokomos. 2017; 28(3): 135-141.

18. Mena Aragón E, Sánchez Sarduy M, Reyes Quintana Y, García Martín D. Effectives of an educational intervention about care-givers' knowledge for preventing mistreatment of the aged adult. Rev Cubana Enfermer. 2016; 32(2): 237-245. 
19. Fernández Daza MP, Martin Carbonell M. Manual básico para la evaluación psicológica de adultos mayores institucionalizados y sus familias. 2017.

20. Torres Prados M, Estrella González IM. Sensibilización y detección del maltrato en el anciano. Hacia una atención primaria adaptada a los mayores. Gerokomos. 2015;26(3): 79-83. doi: http:// dx.doi.org/10.4321/S1134-928X2015000300002

21. Restrepo González LM, Aguirre Escobar JP. Caracterización de un grupo de adultos mayores no institucionalizados: percepción de calidad de vida, estado mental y del ánimo. Universidad de Antioquia. 2018.

22. Llanes Torres HM, López Sepúlveda Y, Vásquez Aguilar JL, Hernández Pérez R. Factores psicosociales que inciden en la depresión del adulto mayor. Revista de ciencias médicas de Mayabeque. 2015; 21(1).

23. Jorquera Flores C, Jorquera Flores S. Los cuidados formales que reciben los adultos mayores en establecimientos de larga estadía: Aplicación del enfoque de derechos. Solonik. 2018; 1(3).

24. Gámez. C.M, Guardián A. Incidencia del abandono de la familia en la situación socio afectiva del adulto mayor en el Asilo de ancianos de la ciudad de León. Nicaragua; 2014.

25. Agudelo Cifuentes MC, Cardona Arango D, Segura Cardona A, Muñoz Rodríguez D, Restrepo Ochoa A. Características sociales y familiares asociadas al maltrato al adulto mayor de Pasto, Colombia 2016. Rev CES Psico. 2019; 12(1): 32-42. doi: https://doi. org $/ 10.21615 / 4387$

26. Torres. M. T, Estrella. M. I. Sensibilización y detección del maltrato en el anciano: hacia una atención primaria adaptada a los mayores. Gerokomos. 2015; 26(3): 79-83. doi: http://dx.doi. org/10.4321/S1134-928X2015000300002

27. Martínez Moreno E, Bermúdez Pérez MP. Maltrato psicológico hacia los mayores: variables a tratar. RECS. 2016; 7(1). doi: http://dx.doi.org/10.20318/ recs.2016.3161

28. Giraldo Osorio A, Vélez Álvarez C, Berra S, Perdomo Rubio A. Calidad de los instrumentos que valoran las funciones de la atención primaria: revisión sistemática. Hacia Promoc Salud. 2020; 25 (1): 109-129. doi: https://doi.org/10.17151/ hpsal.2020.25.1.8

29. Bejines. M, Velasco. R GL, Barajas. A, Aguilar. L RML. Valoración de la capacidad funcional del adulto mayor residente en casa hogar. Rev Enfermería del Inst Mex del Seguro Soc. 2015; 23(1): 9-15.
30. Zapata. B DN, Cardona. D. Apoyo social y familiar del adulto mayor del área urbana. Angelópolis, Antioquia 2011. Rev Salud Pública. 2016; 17(6): 848-860. doi: https://doi.org/10.15446/rsap. v17n6.34739

31. Cardona Arango D, Segura Cardona A, Segura Cardona A, Muñoz Rodríguez DI, Agudelo Cifuentes MC. La felicidad como predictor de funcionalidad familiar del adulto mayor en tres ciudades de Colombia. Hacia Promoc Salud. 2019; 24(1): 97-111.

32. Torres Prados MT, Estrella González IM. Sensibilización y detección del maltrato en el anciano: hacia una atención primaria adaptada a los mayores. Gerokomos. 2015;26(3): 79-83. doi: http:// dx.doi.org/10.4321/S1134-928X2015000300002

33. Mena Aragón E, Sánchez Sarduy M, Reyes Quintana Y, García Martín D. Effectives of an educational intervention about care-givers' knowledge for preventing mistreatment of the aged adult. Rev Cubana Enfermer. 2016; 32(2): 237-245.

34. Carrasco Jiménez E. Incriminación del maltrato corporal relevante y de la sumisión a trato degradante de personas con déficit corporal o físico en la legislación penal chilena. Ley ${ }^{\circ} 21.013 \mathrm{de}$ 2017. Rev Derecho (Concepción). 2018; 86(243), 57-69. doi: http://dx.doi.org/10.4067/S0718591X2018000100057

35. Riveros C, Contreras C, Palomo R. (2018). Mediación y personas mayores en Chile. Rev Facultad de Derecho. 2018; (45): 292-317. doi: http://dx.doi.org/10.22187/rfd2018n45a7

36. Grimaldo Salazar SP, Mancera Ortiz LF. Mental health and cognitive impairment of elderly adults from the municipality of Girardot Colombia. Conrado. 2019; 15(70): 460-464.

37. Fuentes Reyes, Flores Castillo FD. Elderly people homelessness like consequence of abandonment in the state of Mexico. Papeles Población. 2016; 22(87), 161-181.

38. Mier Sosa F. Social violence towards older adults in hypermodernity: Approach from clinical sociology. Psicol Conoc Soc. 2016; 6(2): 188-208.

39. Mora Quezada JN, Osses Paredes CF, Rivas Arenas SM. Funcionalidad del adulto mayor de un Centro de Salud Familiar. Rev Cubana Enfermer. 2017; 33(1): 18-30.

40. Condeza AR, Bastías G, Valdivia G, Cheix C, Barrios $\mathrm{X}$, Rojas R, et al. Elderly in Chile: Describing their needs for preventive health communication. Cuadernos Info Enfermer. 2016; 33(1): 18-30. 
41. Agudelo Cifuentes MC, Cardona Arango D, Segura Cardona A, Restrepo Ochoa DA. Elder abuse, a silent problem. Rev Fac Nac Salud Pública. 2020; 38(2): e331289. doi: https://doi.org/10.17533/udea. rfnsp.e331289

42. García Artuñedo C, Ramos Monteagudo AM, Revelo Álvarez P. Historia de vida de un adulto mayor institucionalizado en un hogar de ancianos en Camagüey, Cuba. Rev Univ Desarrollo Social. 2017; 8(1).

43. Gamba Rincón AP, Páez Martínez GJ, Domínguez Pérez WJ, Rincón Lozada CF. Desempeño neuropsicológico en adultos mayores. Arch Neurocien. 2017; 22(4).

44. Cardona Arango D, Segura Cardona A, Garzón Duque M, Cano Sierra M. Estado de salud del adulto mayor de Antioquia, Colombia. Rev Bras Geriatr Gerontol. 2016; 19(1): 71-86. doi: https:// doi.org/10.1590/1809-9823.2016.14199

45. Ruelas G, Pelcastre RH. Maltrato institucional hacia el adulto mayor: percepciones del prestador de servicios de salud y de los ancianos. Mexico; 2014.

46. Montero G, Vega HG. Abuso y Maltrato en el Adulto Mayor. Vol. 34, Medicina Legal de Costa Rica. Asociación Costarricense de Medicina Forense; 2017; 120-130 p.

47. Cerón M.A. Modelo de atención en salud integral para ancianos que acuden a los asilos de la ciudad de Ibarra, previo diagnóstico. Rev Univ Tec Norte. 2017.

48. Bentancor D. El abuso patrimonial en el viejo desde la sociedad del siglo XXI. ¿Lo urgente no deja tiempo para lo importante? En: XVII Jornadas de Investigación: a 70 años de la Declaración Universal de Derechos Humanos ¿libres e iguales? Montevideo, setiembre 2018. Montevideo: Udelar. FCS. 2018.

49. Sotomayor Álvarez M, Bermúdez Lusa G, Pérez Díaz R, Méndez Amador T. Maltrato en personas mayores. Gerolnfo. 2015; 10(3).

50. Muñoz Lara J. Depresión en la persona adulta mayor institucionalizada: Un nuevo reto. RHCS. 2015; 1(1): 68-69.

51. Martínez Álvarez N. Estudio de caso en Hogar de Ancianos "El Ceibo" en la ciudad de Young: las representaciones que tienen los adultos mayores institucionalizados acerca de los vínculos con su familia. Universidad de la republica Uruguay. 2018.

52. Serra Valdés M, Cordero López G, Viera García M. El maltrato a los ancianos o el no mejor trato. Realidad y retos. Medisur. 2018; 16(2): 233-240.
53. Varela Pinedo LF. Salud y calidad de vida en el adulto mayor. 2016; 33(2). doi: https://doi.org/10.17843/ rpmesp.2016.332.2196

54. Acosta Quiroz CO, Tanori Quintana J, García Flores R, Echeverría Castro SB, Vales García JJ, Rubio L. Soledad, depresión y calidad de vida en adultos mayores mexicanos. Psicol Salud. 2017; 27(2): 179188.

55. Riveros C, Rodríguez P, Palomo R, Alvear S, Fernández MA, Arenas A. El maltrato estructural a personas mayores en chile y la necesidad de formular un índice multidimensional. Universum. 2017; 32(2): 163-176. doi: http://dx.doi.org/10.4067/ S0718-23762017000200163

56. Balseca Basantes J, Arcos Bombon E. Relaciones familiares y abandono del adulto mayor institucionalizado en el hogar Sagrado Corazón de Jesús de la Ciudad de Ambato. 2017.

57. Sarmiento Fernández AM, Escobar Bueno, CM. Calidad de vida en adultos mayores institucionalizado y no institucionalizados de la ciudad de Bucaramanga y su área Metropolitana [trabajo de grado]. 2018.

58. Álvarez Vega M, Cortes Vadilla MV, Quirós Mora LM. Abuso en el adulto mayor: una situación que va en aumento. 2020; 5(6): e514 doi: https://doi. org/10.31434/rms.v5i6.514 